\title{
EL PROBLEMA DE LA EQUIVALENCIA LÓGICA EN LA PARADOJA DE LOS CUERVOS
}

Durante los últimos veinticinco años se ha discutido mucho la Paradoja de los Cuervos, conocida también como la Paradoja de la Confirmación. Esta Paradoja, formulada por Carl Hempel y otros, encierra importantes problemas teóricos relativos a la inducción y a la deducción. El enunciado de la paradoja es muy simple. Se deriva de tres proposiciones de la siguiente manera: ${ }^{1}$

Prop. (1) Observaciones sobre cuervos negros confirman "Todos los cuervos son negros."

Prop. (2) Observaciones sobre otras cosas que no son cuervos, sean o no negras, no confirman "Todos los cuervos son negros."

Prop. (3) Si las observaciones confirman una formulación de una hipótesis, entonces confirman cualquier formulación lógicamente equivalente.

Ahora bien, es muy fácil mostrar que estas tres proposiciones son incompatibles unas con otras. Obviamente "Todo lo no-negro es no-cuervo" es lógicamente equivalente a "Todos los cuervos son negros." Y puesto que una observación sobre un cisne blanco es una observación de un no-cuervo no-negro se sigue, por el criterio implícito en la Prop. (1), que una observación sobre un cisne blanco confirma "Todos los cuervos son negros." Pero en base a la Prop. (2), observaciones sobre cisnes blancos no confirman "Todos los cuervos son negros."

No cabe duda de que si se consideran juntas, las Prop. (1) y (2) expresan el criterio empírico que dice que para que una hipótesis pueda confirmarse mediante observación directa, las cosas a las que se refieren los términos de la hipótesis deben observarse directamente. Las Props. (1) y (2), tomadas conjun-

1 Las formulaciones de las Proposiciones (1) y (3) dadas aquí están tomadas del artículo de J. L. Mackie "La Paradoja de la Confirmación", The British Journal for the Philosophy of Science, Feb. 1963, 13 p. 265. La formulación de la proposición (2) la sugerí en mi artículo "Confirmación mediante observación y la Paradoja de los Cuervos", The Bristih Journal for the Philosophy of Science, Nov. 1964, 15, pp. 200-212. 
tamente, pueden, por tanto, considerarse como una definición de la relación "confirmación mediante observación directa" que puede darse entre las observaciones y una hipótesis.

Ahora bien, ciertamente no puede negarse que el concepto de esta relación es un concepto aceptado en la ciencia empírica. Claro está que no es el concepto de todo el proceso de confirmación empírica de los enunciados científicos, sino más bien el concepto de la confirmación en cuanto tal de un tipo particular de enunciado: a saber, la confirmación, mediante observación directa, de una hipótesis observacional. Por otra parte, es perfectamente posible definir esta relación de confirmación en una forma ambigua, como en efecto lo hacen las Props. (1) y (2) tomadas conjuntamente. Por tanto, ya sea que se considere o no que el concepto de esta relación es un concepto científicamente útil, el hecho es que este concepto es un criterio metodológico que puede usarse. $\mathrm{Y}$, de esta manera, si el científico desea usarlo, es difícil ver por qué no podría hacerlo. Sin embargo, la Prop. (3) aduce una consideración lógico formal que tiene la consecuencia de que el concepto que es definido por las Props. (1) y (2), tomadas conjuntamente, no es lógicamente admisible. Porque en la Prop. (3) se sostiene implícitamente que, dada cualquier relación que tiene a una proposición como uno de sus términos, si se da la relación con respecto a una proposición dada, entonces también debe darse con respecto a todas las formulaciones lógicamente equivalentes. No se sostiene esto a la ligera. Porque si, como se ha supuesto comúnmente, las proposiciones lógicamente equivalentes son incondicionalmente intercambiables unas con otras, entonces la Prop. (3) debe ser válida. Si la Prop. (3) no es válida, esto significa que las proposiciones lógicamente equivalentes no son incondicionalmente intercambiables unas con otras, y esto parece amenazar uno de los supuestos más fundamentales de la inferencia deductiva. Si concedemos, por otra parte, la validez de la Prop. (3), entonces esto expone a un concepto aceptado, que pertenece a la metodología de la ciencia empírica, a un veto por parte de la lógica formal. La seriedad del dilema es así evidente.

La mayoría de las personas que han discutido la paradoja han asumido que la Prop. (3) es incuestionablemente válida. Y puesto que usualmente se considera que el problema no es el de la confirmación conclusiva, sino. sólo el de un cierto poder confirmatorio, no ha habido mucha preocupación en relación a la Prop. (1). La atención se ha concentrado, más bien, en la posibilidad de rechazar la Prop. (2). Algunos han argumentado que en relación a "Todos los cuervos son negros" es posible considerar el poder confirmatorio de las observaciones de cuervos negros y de no-cuervos no-negros, respectivamente, en un contexto acerca del cual hay alguna información previa. Esto es, cierta información acerca del número de cuervos y de no-cuervos hace posible llegar a una estimación del relativo poder confirmatorio de tales observaciones. Otros han aducido el poder con- 
firmatorio de casos comprobatorios en los que observaciones de no-negros que podrian ser cuervos resultan ser no-cuervos.

Ahora bien, ambos tipos de argumento recurren, en forma evidente, a una redefinición de la confirmación. Esto es, se concede que el concepto de confirmación definido por las Props. (1) y (2) no es lógicamente admisible; y de esta manera el problema de la paradoja parece convertirse en el de volver a definir la confirmación en una forma tal que sea lógicamente admisible. Así, cuando se acepta la Prop. (3), esto parece ser una invitación a que el argumento se ocupe con el eterno problema de la confirmación de las generalizaciones inductivas hechas por enumeración de casos. Me parece, sin embargo, que la verdadera cuestión de la paradoja no es el problema empírico de definir la confirmación observacional, sino más bien el de si el específico y bien establecido concepto de confirmación mediante observación directa, tal como se define en las Props. (1) y (2), es un concepto lógicamente posible.

La Prop. (3) parece descansar en la tesis de que cualquier relación que tenga una proposición como uno de sus términos, debe ser compatible con la intercambiabilidad incondicional de las proposiciones lógicamente equivalentes. $\mathrm{Y}$ de este modo, si la definición de una relación de confirmación cualquiera es incompatible con esa intercambiabilidad, entonces esa definición no es lógicamente admisible. La tesis que propuse en mi artículo anterior, y que deseo desarrollar aquí, es la de que esta consecuencia radical de la Prop. (3) ha sido aceptada con demasiada ligereza. Claro está que las soluciones usuales de la paradoja logran tomar en cuenta la eficacia, en la práctica, del criterio empírico de confirmación observacional directa. Esto es, ellos conceden, en relación a "Todos los cuervos son negros", el poder confirmatorio prácticamente nulo de observaciones sobre objetos blancos misceláneos. Pero el punto importante que conceden es el de que una observación acerca de un cisne blanco tiene necesariamente algún poder confirmatorio y, por consiguiente, rechazan la Prop. (2) que expresa la consecuencia del concepto de confirmación mediante observación directa, a saber, que una observación acerca de un cisne blanco no tiene poder confirmatorio en relación a "Todos los cuervos son negros." De esta manera renuncian en principio a la legitimidad del concepto que el científico empírico tiene del criterio de la confirmación observacional directa de una hipótesis.

Parecería que se pretende justificar esto por el hecho de que las hipótesis son proposiciones y, en cuanto tales, pertenecen al dominio de la lógica formal, de manera tal que cuando el científico empírico habla acerca de proposiciones debe obedecer las reglas lógicas formales que rigen para ellas. De pronto esto parece bastante plausible. Sin embargo, cuando el lógico se toma el trabajo de insistir - como frecuentemente hace- en que el rechazo de la Prop. (2) realmente no va en contra de la intuición, se tiene la impresión de que tácitamente está concediendo que, por lo que se ve, la Prop. (2) parece ser perfectamente inteligible. $\mathrm{Y}$ esto es lo que hace que todo el problema sea tan confuso. Porque 
nosotros estamos presos en el dilema de que en tanto que la Prop. (2) no ofrece en sí misma ninguna dificultad conceptual sino que, por el contrario, es perfectamente aceptable, se nos pide, sin embargo, que la rechacemos por razones a priori. Parece, entonces, que aquí algo anda mal. Porque si el concepto de equivalencia lógico formal es incompatible con un concepto metodológico de la ciencia empírica que es absolutamente claro, entonces el rechazo del último concepto para salvar el primero sin duda no resuelve la dificultad. Esto, en verdad, sólo hace que el problema se vuelva más opaco, porque supone el recurso a la tesis, desde hace mucho desacreditada, de que las consideraciones a priori pueden, en algún sentido, determinar el contenido de las aserciones hechas en un dominio empírico, en este caso el dominio de la metodología de la ciencia empírica misma. Conceder por razones a priori que la confirmación observacional tal vez no es definida por las Props. (1) y (2), aun cuando esta definición suministra un concepto claro de un tipo particular de procedimiento científico es, sin duda, una solución muy insatisfactoria de la dificultad teórica. Si nosotros, por otra parte, rechazamos la Prop. (3) no podemos evitar encontrarnos con el problema de cuáles sean las consecuencias que esto implica para el concepto de equivalencia lógica. En mi artículo anterior rechacé la Prop. (3) sobre la base de que la relación empíricamente definida de la confirmación observacional directa sencillamente no es transitiva con respecto a todas las proposiciones lógicamente equivalentes. En lo que aquí sigue quisiera sugerir que el rechazo de la Prop. (3) es compatible con el concepto de equivalencia lógico formal. $\mathrm{Mi}$ argumento establecerá dos tesis principales: en primer lugar, que la equivalencia lógica no significa necesariamente igualdad y que, por consiguiente, no hay razones a priori parà asumir la intercambiabilidad incondicional de las expresiones lógicamente equivalentes; y, en segundo lugar, que el uso de expresiones de equivalencia lógica como principio de inferencia no se aplican al caso de la confirmación. Apelando a una distinción establecida por Susanne Langer es fácil mostrar que, en el caso de la confirmación, una proposición aparece como un término de la relación constituyente, más bien que como un término de una relación lógica; de manera que este caso no afecta la intercambiabilidad de proposiciones lógicamente equivalentes cuando éstas aparecen como términos de relaciones lógicas en una secuencia de razonamiento formal.

Antes de discutir estas dos tesis en forma detallada, desearía, primero, discutir en una forma general por qué pienso que son relevantes al problema de la Paradoja de los Cuervos.

La Prop. (3) dice que si las observaciones confirman cualquier miembro de un conjunto de proposiciones lógicamente equivalentes, entonces confirman todos los miembros del conjunto. Esta tesis se propone como si fuese evidente- 
mente válida. Sin embargo, las razones en las que se apoya no son de ninguna manera claras.

El problema de los fundamentos de la Prop. (3) puede ponerse de manifiesto mediante la siguiente consideración: Cada una de las tres proposiciones que constituyen la paradoja afirma algo acerca de una relación de confirmación. Sin embargo, cada una de estas tres proposiciones no afirma algo acerca de la misma relación de confirmación. Tomadas en conjunto, las Props. (1) y (2) definen el concepto de la relación de confirmación observacional directa. Éste es un concepto muy específico que tiene un significado que es comprendido por todos. La Prop. (3), por otra parte, afirma una condición definitoria de cualquier concepto de cualquier relación de confirmación que pueda darse entre observaciones y una hipótesis. Las Props. (1) y (2) especifican, por consiguiente, las propiedades definitorias de una específica relación de confirmación concebida empíricamente, en tanto que la Prop. (3) afirma lo que se pretende que es una propiedad a priori de cualquier posible relación de confirmación.

La cuestión es, por tanto, la de saber en qué se apoya la Prop. (3) para proponer esta tesis a priori. Esto es ¿cuáles son las razones para sostener que la intercambiabilidad de proposiciones lógicamente equivalentes es una condición a priori de la relación de confirmación en general? Si se pretendiera que el concepto de equivalencia lógico formal significa la igualdad de todas las expresiones lógicamente equivalentes, entonces se seguiría que esas expresiones equivalentes son intercambiables en todas las circunstancias y esto incluiría, desde luego, el caso de cualquier relación de confirmación. Sin embargo, no es de ninguna manera evidente que el concepto de equivalencia lógico formal significa que dos expresiones equivalentes cualesquiera sean necesariamente la misma. Pero si las expresiones lógicamente equivalentes no son necesariamente las mismas expresiones, entonces su mutua intercambiabilidad debe basarse en alguna otra razón. Por de pronto en el uso que comúnmente se hace del concepto de equivalencia lógica, este concepto parece aplicarse peculiarmente al razonamiento formal acerca de las relaciones lógicas entre proposiciones. Que este concepto pueda tener ese dominio peculiar de aplicación, sólo lo proponemos aquí, desde luego, como una sugerencia. Todo lo que deseo sostener es que si puede mostrarse que equivalencia lógica no significa igualdad y si también puede mostrarse que hay un tipo de situación lógica en la que el intercambio de proposiciones lógicamente equivalentes no es siempre válido, entonces esto sugiere que dicho intercambio no es un procedimiento universalmente válido, sino, más bien, un procedimiento cuya validez se limita a un dominio definible.

Desde este punto de vista, entonces, estaríamos justificados en preguntar en qué se apoya la Prop. (3) para sostener que la relación de confirmación pertenece al dominio en el cual el intercambio de proposiciones lógicamente equivalentes es siempre válido. Porque muy bien podría suceder que la relación de confirmación perteneciera al dominio en el que dicho intercambio no es válido. 
3

Por lo general se está de acuerdo en que la equivalencia lógič́a de dos expresiones significa que pueden recíprocamente inferirse la una de la otra, o de que cada una de ellas implica la otra. Que esto incluya o no el caso en que se reitera la afirmación de la misma proposición depende, por tanto, de cómo se defina la inferencia. Porque si aquí estamos hablando de la inferencia en cuanto tal y la definimos como significando el paso justificado de una o más proposiciones a una proposición diferente, entonces este concepto de inferencia es incompatible con la noción de inferir la misma proposición a partir de sí misma. Pero si, en cambio, estamos aqui hablando del proceso de inferencia más amplio en el curso del cual podemos afirmar cualquier proposición que se sigue válidamente de una - más proposiciones dadas, entonces la equivalencia lógica puede interpretarse como incluyendo la afirmación reiterada de la misma proposición. Ahora bien, me parece que la cuestión de cuál sea el concepto de inferencia del que hablamos es irrelevante con respecto a la cuestión de si el concepto de equivalencia lógica significa igualdad. Porque aún si estamos hablando de este último concepto, que incluye la reiterada afirmación de la misma proposición, —entonces en virtud de este concepto- algunas expresiones lógicamente equivalentes serían la misma expresión, en tanto que otras expresiones equivalentes serían expresiones diferentes. Mi posición es que lo que aquí es problemático no es si expresiones lógicamente equivalentes son siempre las mismas, sino si alguna vez son. la misma. Porque evidentemente no son siempre la misma.

Obviamente, no hay necesidad de insistir sobre esto. Es muy fácil, por ejemplo, citar expresiones lógicas que son lógicamente equivalentes y que difieren con respecto a su forma lógica. Sin duda $\sim(p \cdot q)$ y $\left(\sim p^{v} \sim q\right)$ no tienen la misma forma $y$, sin embargo, son lógicamente equivalentes. Es obvio, en verdad, que la utilidad del concepto de equivalencia lógica consiste precisamente en la intercambiabilidad de expresiones diferentes. Pero si esto es así, entonces la equivalencia lógica no significa igualdad. Se sigue, por consiguiente, que la intercambiabilidad de expresiones lógicamente equivalentes no puede basarse en su igualdad, sino más bien en la posibilidad de que puedan inferirse la una de la otra. Si pudiera mostrarse que dos expresiones dadas son la misma, entonces se seguiría su intercambiabilidad incondicional. Pẹro si puede mostrarse que dos expresiones semejantes son diferentes, entonces lo más que tal vez puede decirse es que su equivalencia lógico formal es la base de su intercambiabilidad lógico formal. Además, si expresiones equivalentes no son siempre iguales, entonces en cada caso hay que determinar si son o no iguales.

En el caso de las proposiciones "Todos los cuervos son negros" y "Todo lo no-negro es no-cuervo", la diferencia entre ellas tiene cuando menos tres aspectos relacionados entre sí. En primer lugar, son diferentes en la forma lógica. En 
segundo lugar, sus términos se refieren a cosas diferentes. En tercer lugar, todas las relaciones no son transitivas con respecto a ellas.

En lo tocante a su forma lógica ambas son, claro está, proposiciones $A$ tradicionales. Pero debe tenerse presente que en la inferencia inmediata tradicional, las proposiciones lógicamente equivalentes pueden ser de diferentes formas tradicionales, como, por ejemplo, $A$ y $E$. Además, en el caso de las contrapositivas, en que partimos de una forma $A$, es necesario pasar por la forma $E$ para poder inferir una proposición equivalente que esté en la forma $A$. Pero lo que tiene aquí un interés mayor es que el hecho de que los términos de la proposición contrapositiva deducida sean positivos o negativos depende de las propiedades formales de la equivalencia, ya que los términos de la proposición contrapositiva deducida son los contradictorios de los términos de la proposición original. Así, en tanto que estas dos proporciones son de la forma $A$, hay sin embargo una diferencia formal entre ellas con respecto de sus términos. Los términos no pueden ser los mismos en ambas proposiciones.

En segundo lugar, la relación formal entre los términos de las dos proposiciones tiene la consecuencia de que los términos de las dos proposiciones se refieren a cosas diferentes. Así las dos distintas proposiciones afirman algo diferente acerca de cosas diferentes. Claro está que lo que una de ellas afirma está implicado por la otra; sin embargo, lo que una de ellas afirma no lo afirma la otra. Esta importante distinción se pone de relieve cuando las respectivas proposiciones se convierten en términos de la relación de confirmación observacional, porque en este caso debe especificarse la referencia de sus términos. Al hacer esto se advierte con claridad que cuando se considera que estas proposiciones tienen alcance existencial "Todo lo no-negro es no-cuervo" hace una afirmación acerca de cosas diferentes a las que son afirmadas por la proposición "Todos los cuervos son negros." Esto mismo puede verse considerando las formas "Si $x$ es un cuervo, entonces $x$ es negro" y "Si $x$ es no-negro, entonces $x$ es un no-cuervo", porque inmediatamente vemos que el mismo valor no puede sustituirse por $x$ en ambos casos. Esta consideración hace ver que una relación de confirmación implica la referencia de términos específicos. Así, cuando una proposición pasa a ser un término de esta relación, no llega a serlo por ser una expresión de un "estado de cosas" el cual es descrito por un conjunto de proposiciones lógicamente equivalentes, sino más bien por ser una expresión de una cierta parte de un "estado de.cosas". Y sería sin duda muy extraño si hubiese alguna razón a priori por la cual estos términos de relaciones no debieran ser lógicamente admisibles.

Es evidente, desde luego, que los términos de "Todos los cuervos son negros" y "Todo lo no-negro es no-cuervo" se refieren a cosas diferentes. Si se ha asumido que éstas son formulaciones verbalmente diferentes de la misma proposición, esta suposición se ha hecho a despecho de esa obvia diferencia entre ellas. Tal vez se ha supuesto en forma tácita que, lógicamente, lo que afirma una proposición 
incluye todo lo que inmediatamente implica. Pero, sin duda, esto es pasar por alto el hecho de que si una inferencia inmediata tiene el poder de pasar de una afirmación acerca de un tipo de cosas a una afirmación acerca de otro tipo de cosas, esto es sólo porque una inferencia semejante está justificada por un principio de inferencia válido. Lo que aquí está a discusión es que nunca podemos dar un sólo paso más allá de lo que una proposición directamente afirma a lo que dicha proposición implica sin introducir los fundamentos lógicos de la implicación. Me parece que esto debe concederse sea cual fuere la posición filosófica que se adopte frente a estos fundamentos lógicos. Cilaro está que podría argumentarse que es evidente que "Todo lo no-negro es no-cuervo" es equivalente a "Todos los cuervos son negros"; pero si esto es evidente, sólo lo es porque los principios de inferencia en cuestión son evidentes. Porque no puede negarse que la noción de negación, así como algunas nociones de relaciones de clases, ha sido introducida. Me parece que ya sea que los fundamentos lógicos de la conversión y obversión tradicionales sean o no absolutamente explícitos y evidentes, todos conceden que hay una línea de razonamiento que lleva de "Todos los cuervos son negros" a su contrapositivo, y que este razonamiento es válido. $\mathrm{Y}$ ésta es la razón por la cual estamos tan seguros de que estas dos proposiciones son lógicamente equivalentes a pesar de las diferencias entre ellas.

En tercer lugar, la diferencia entre estas dọ proposiciones con respecto a la referencia de sus términos, tiene la consecuencia de que la relación de confirmación, tal como está definida por las Props. (1) y (2), no es transitiva de una de estas proposiciones a la otra. Esto es, si las observaciones mantienen esta relación con una de estas hipótesis, no la mantienen con las otras. Es muy importante ver, además, que esto es sumamente claro. $\mathrm{O}$ sea, el concepto empírico de la confirmación observacional directa que se expresa en las Props. (1) y (2), es completamente definido. La regla dice, simplemente, que para que una observación pueda confirmar una hipótesis, lo que se observa debe ser una instancia de lo que se enuncia en la hipótesis. Esto significa que las cosas a las que se refieren los términos de la hipótesis deben observarse directamente. Si esta condición no se cumple, entonces no tenemos una instancia de confirmación observacional directa. Tampoco está viciada esta regla por el hecho de que a menudo es necesario que el observador especifique que sus observaciones son sobre ciertas manifestaciones, por ejemplo; "un resplandor sobre una pantalla", más bien que sobre "un electrón mismo". Casos semejantes, en verdad, sirven para ilustrar la necesidad de distinguir entre las hipótesis observacionales formuladas con precisión y algunas otras hipótesis que se consideran como si fuesen confirmadas o no confirmadas por el resultado de la observación en base a una cadena de razonamiento que conecta las dos hipótesis. Porque es precisamente el mantener esta distinción entre lo que de hecho se confirma mediante la observación y lo que subsecuentemente se infiere, lo que garantiza que los esquemas conceptuales tradicionales, que son la base de las inferencias subsecuentes, estén 
sujetos al análisis crítico cuando resultan inadecuados para habérselas con los resultados de las observaciones. ${ }^{2}$ Desde este punto de vista las proposiciones (1) y (2) pueden interpretarse como definiendo las condiciones de las hipótesis observacionales confirmables, precisamente porque estas proposiciones son condiciones definitorias de una relación que tiene esas hipótesis como uno de sus términos.

El concepto de esta relación es, por tanto, claro, y es posible determinar sus propiedades. Que esta relación sea transitiva con respecto a todas las proposiciones lógicamente equivalentes, depende tanto de la naturaleza de esta relación como de la naturaleza de esas proposiciones. Si, entonces, la naturaleza de la relación misma es tal que dadas las proposiciones "Todos los cuervos son negros" y "Todo lo no-negro es no-cuervo", la relación no puede ser transitiva de la una a la otra, entonces esto mismo demuestra que esas proposiciones son diferentes. Porque si fueran la misma proposición, entonces siempre que una de ellas apareciera como el término de una relación cualquiera, la otra seria intercambiable con ella.

Mi tesis aquí es la de que la relación de confirmación observacional directa forma parte del enunciado de esta paradoja como una relación dada, poseyendo su carácter propio que es claramente definido por las proposiciones (1) y (2). El hecho de que es una relación definida que pertenece al método inductivo da pie a la ilusión de que en esta paradoja se discute su naturaleza; pero este no es el caso. Me parece, en verdad, que la noción de que las propiedades de esta relación pueden determinarse por una consideración formal, es la falacia central de la paradoja. Porque es exactamente lo contrario. Esto es, si la teoría formal de la equivalencia lógica no fuese compatible con el concepto, claramente concebido, de confirmación observacional directa, esto más bien obligaría a que se reconsiderara la teoría formal.

Tal vez esta tesis no sería conclusiva si la relación de confirmación observacional directa fuese el único caso que muestra que las proposiciones lógicamente equivalentes no son incondicionalmente intercambiables. Pero este caso es sólo una consecuencia del hecho de que las proposiciones pueden aparecer como los términos de varias relaciones que no son transitivas con respecto a todos los equivalentes lógicos. La relación de "formular" es ella misma un buen ejemplo de lo anterior. Si decimos "El científico $x$ formuló la hipótesis $H$ ", esto sin duda significa que cualquier valor de $H$ es una determinada formulación, y no cualquier formulación que pertenezca a un conjunto de equivalentes lógicos. En otras palabras, el hecho de que las "formulaciones" de hipótesis lógicamente equivalentes son formalmente diferentes y de que guardan ciertas relaciones formales las unas con las otras es, en sí mismo, la prueba más clara de que las proposiciones lógicamente equivalentes no son incondicionalmente intercambiables las

2 Esta tesis está desarrollada más ampliamente en mi anterior artículo —al que me referí antes-- sobre esta paradoja. 
unas por las otras. Porque las relaciones "ser el converso de", "ser el contrapositivo de", etc., pueden citarse como el caso paradigmático de un tipo de relación que no es transitiva con respecto a todas las proposiciones lógicamente equivalentes. Tampoco me parece que este argumento pueda refutarse aduciendo que recurre a la auto-referencia. Claro está que recurre. Pero este argumento sólo es posible en razón de la diferencia entre proposiciones lógicamente equivalentes.

En lo que toca al problema específico de la relación de confirmación, podemos considerar la siguiente proposición:

"Si un concepto dado es un término de un miembro de un conjunto de proposiciones lógicamente equivalentes, entonces éste es un término de cualquier proposición que pertenezca al conjunto."

Obviamente, esto es falso. La relación "ser un término de" no es transitiva con respecto a todas las proposiciones lógicamente equivalentes. $\mathrm{Y}$ precisamente esta es la razón por la cual la relación de confirmación observacional, que supone la referencia de los términos, no'es transitiva de "Todo lo no-negro es no-cuervo" a "Todos los cuèrvos son negros".

Desearía ahora tratar de sugerir, en una forma muy tentativa, por qué la intercambiabilidad de expresiones lógicamente equivalentes en un razonamiento formal no se afecta por el rechazo de la proposición (3) en la Paradoja de los Cuervos.

En cada una de las proposiciones que constituyen la Paradoja, una proposición aparece como un término de una relación, a saber, una dada relación de confirmación. Pero esta relación es un constituyente más bien que una relación lógica en el sentido especificado por Susanne Langer, quien lo formula de la siguiente manera:

"Una relación que pertenece al contexto formal, la llamo una relación constituyente del discurso. Es uno de los conceptos que se asumen explícitamente como un constituyente legítimo de las proposiciones en ese discurso..." 3

Dichas relaciones se dan entre los elementos del discurso. Ellas

"...varían según el contexto formal; en todo discurso debe haber relaciones constituyentes, pero cuáles deben ser éstas, es arbitrario dentro de los límites de lo que 'tiene sentido' en un universo determinado... Las relaciones que se dan entre los elementos forman proposiciones elementales y son constituyentes de esas proposiciones e ítems en el contexto formal..." Por otra parte,

3 An Introduction to Symbolic Logic, $2^{4}$ ed., Dover, N. Y., p. 70. 
“... las relaciones que se dan entre proposiciones no son constituyentes de proposiciones elementales y, por tanto, no forman parte de los materiales del contexto formal. A este último tipo lo llamaré relaciones lógicas..." 4

Por relaciones lógicas la Señora Langer no entiende esas relaciones entre proposiciones como "converso de" o "contrapositivo de" (que más bien parecerían ser relaciones constituyentes de proposiciones acerca de proposiciones), sino más bien las constantes lógicas: conjunción, implicación, etc.

Sobre la base de esta distinción, me parece que sería posible decir que las expresiones lógicamente equivalentes son intercambiables las unas por las otras cuando aparecen como los términos de relaciones lógicas (esto es, las constantes lógicas), pero que no son necesariamente intercambiables cuando aparecen como los términos de relaciones constituyentes. Porque en este último caso funcionan lógicamente como término de relaciones constituyentes; y su intercambiabilidad se determina, por consiguiente, en el contexto de las propiedades definidas de las relaciones constituyentes.

Elaborar esta sugerencia rebasa los fines de este artículo. Me parece, sin embargo, que es claro que la relación de confirmación observacional directa definida empíricamente, es una relación constituyente en el sentido definido por la Señora Langer. Y puesto que sus propiedades son tales que ella no es transitiva con respecto a todas las proposiciones lógicamente equivalentes, ésta es una condición limitativa de la intercambiabilidad de esas proposiciones cuando una de ellas aparece como un término de la relación. Es interesante advertir que cuando una proposición funciona lógicamente como un término de esta relación, se habla de ella como de una hipótesis. Esto pone de relieve que está cumpliendo una función especial como un constituyente de una proposición, y que esta función es diferente de la que cumple cuando es un término de una relación lógica. Por consiguiente parece plausible pensar que aun cuando las proposiciones pueden tener entre sí relaciones constituyentes y su intercambiabilidad con sus equivalentes lógicos puede, por tanto, limitarse debido a las propiedades de esas relaciones, sin embargo esto no afecta a su intercambiabilidad cuando aparecen como los términos de relaciones lógicas.

\section{Judith SGHoenberg}

(traducción de Alejandro Rossi)

4 Op. Cit., p. 74. 